Ana I. Pereira - Florbela P. Fernandes .

João P. Coelho · João P. Teixeira .

Maria F. Pacheco · Paulo Alves ·

Rui P. Lopes (Eds.)

Optimization, Learning

Algorithms and Applications

First International Conference, OL2A 2021

Bragança, Portugal, July 19-21, 2021

Revised Selected Papers

望 Springer 


\section{Editors}

Ana I. Pereira

Instituto Politécnico de Bragança

Bragança, Portugal

João P. Coelho

Instituto Politécnico de Bragança

Bragança, Portugal

Maria F. Pacheco

Instituto Politécnico de Bragança

Bragança, Portugal

Rui P. Lopes (D)

Instituto Politécnico de Bragança

Bragança, Portugal
Florbela P. Fernandes (D)

Instituto Politécnico de Bragança

Bragança, Portugal

João P. Teixeira (1D)

Instituto Politécnico de Bragança

Bragança, Portugal

Paulo Alves

Instituto Politécnico de Bragança

Bragança, Portugal

ISSN 1865-0929

ISSN 1865-0937 (electronic)

Communications in Computer and Information Science

ISBN 978-3-030-91884-2

ISBN 978-3-030-91885-9 (eBook)

https://doi.org/10.1007/978-3-030-91885-9

\section{(C) Springer Nature Switzerland AG 2021}

This work is subject to copyright. All rights are reserved by the Publisher, whether the whole or part of the material is concerned, specifically the rights of translation, reprinting, reuse of illustrations, recitation, broadcasting, reproduction on microfilms or in any other physical way, and transmission or information storage and retrieval, electronic adaptation, computer software, or by similar or dissimilar methodology now known or hereafter developed.

The use of general descriptive names, registered names, trademarks, service marks, etc. in this publication does not imply, even in the absence of a specific statement, that such names are exempt from the relevant protective laws and regulations and therefore free for general use.

The publisher, the authors and the editors are safe to assume that the advice and information in this book are believed to be true and accurate at the date of publication. Neither the publisher nor the authors or the editors give a warranty, expressed or implied, with respect to the material contained herein or for any errors or omissions that may have been made. The publisher remains neutral with regard to jurisdictional claims in published maps and institutional affiliations.

This Springer imprint is published by the registered company Springer Nature Switzerland AG The registered company address is: Gewerbestrasse 11, 6330 Cham, Switzerland 


\section{Preface}

The volume CCIS 1488 contains the refereed proceedings of the International Conference on Optimization, Learning Algorithms and Applications (OL2A 2021), an event that, due to the COVID-19 pandemic, was held online.

OL2A 2021 provided a space for the research community on optimization and learning to get together and share the latest developments, trends, and techniques as well as develop new paths and collaborations. OL2A 2021 had more than 400 participants in an online environment throughout the three days of the conference (July 19-21, 2021), discussing topics associated to areas such as optimization and learning and state-of-the-art applications related to multi-objective optimization, optimization for machine learning, robotics, health informatics, data analysis, optimization and learning under uncertainty, and the Fourth Industrial Revolution.

Four special sessions were organized under the following topics: Trends in Engineering Education, Optimization in Control Systems Design, Data Visualization and Virtual Reality, and Measurements with the Internet of Things. The event had 52 accepted papers, among which 39 were full papers. All papers were carefully reviewed and selected from 134 submissions. All the reviews were carefully carried out by a Scientific Committee of $61 \mathrm{PhD}$ researchers from 18 countries.

July 2021

Ana I. Pereira 


\section{Organization}

\section{General Chair}

Ana Isabel Pereira

Polytechnic Institute of Bragança, Portugal

\section{Organizing Committee Chairs}

Florbela P. Fernandes

João Paulo Coelho

João Paulo Teixeira

M. Fátima Pacheco

Paulo Alves

Rui Pedro Lopes

\section{Scientific Committee}

Ana Maria A. C. Rocha

Ana Paula Teixeira

André Pinz Borges

Andrej Košir

Arnaldo Cândido Júnior

Bruno Bispo

Carmen Galé

B. Rajesh Kanna

C. Sweetlin Hemalatha

Damir Vrančić

Daiva Petkeviciute

Diamantino Silva Freitas

Esteban Clua

Eric Rogers

Felipe Nascimento Martins

Gaukhar Muratova

Gediminas Daukšys

Glaucia Maria Bressan

Humberto Rocha

José Boaventura-Cunha

José Lima

Joseane Pontes

Juani Lopéz Redondo
Polytechnic Institute of Bragança, Portugal

Polytechnic Institute of Bragança, Portugal

Polytechnic Institute of Bragança, Portugal

Polytechnic Institute of Bragança, Portugal

Polytechnic Institute of Bragança, Portugal

Polytechnic Institute of Bragança, Portugal 


Jorge Ribeiro
José Ramos
Kristina Sutiene
Lidia Sánchez
Lino Costa
Luís Coelho
Luca Spalazzi
Manuel Castejón Limas
Marc Jungers
Maria do Rosário de Pinho
Marco Aurélio Wehrmeister
Mikulas Huba

Michał Podpora

Miguel Ángel Prada

Nicolae Cleju

Paulo Lopes dos Santos

Paulo Moura Oliveira

Pavel Pakshin

Pedro Luiz de Paula Filho

Pedro Miguel Rodrigues

Pedro Morais

Pedro Pinto

Rudolf Rabenstein

Sani Rutz da Silva

Sara Paiva

Sofia Rodrigues

Sławomir Stępień

Teresa Paula Perdicoulis

Toma Roncevic

Vitor Duarte dos Santos

Wojciech Paszke

Wojciech Giernacki
Polytechnic Institute of Viana do Castelo, Portugal

NOVA University Lisbon, Portugal

Kaunas University of Technology, Lithuania

University of León, Spain

University of Minho, Portugal

Polytecnhic Institute of Porto, Portugal

Marche Polytechnic University, Italy

University of León, Spain

Université de Lorraine, France

University of Porto, Portugal

Federal University of Technology - Paraná, Brazil

Slovak University of Technology in Bratislava, Slovakia

Opole University of Technology, Poland

University of León, Spain

Technical University of Iasi, Romania

University of Porto, Portugal

University of Trás-os-Montes and Alto Douro, Portugal

Nizhny Novgorod State Technical University, Russia

Federal University of Technology - Paraná, Brazil

Catholic University of Portugal, Portugal

Polytechnic Institute of Cávado e Ave, Portugal

Polytechnic Institute of Viana do Castelo, Portugal

Friedrich-Alexander-University of Erlangen-Nürnberg, Germany

Federal University of Technology - Paraná, Brazil

Polytechnic Institute of Viana do Castelo, Portugal

Polytechnic Institute of Viana do Castelo, Portugal

Poznan University of Technology, Poland

University of Trás-os-Montes and Alto Douro, Portugal

University of Split, Croatia

NOVA University Lisbon, Portugal

University of Zielona Gora, Poland

Poznan University of Technology, Poland 


\section{Contents}

\section{Optimization Theory}

Dynamic Response Surface Method Combined with Genetic Algorithm

to Optimize Extraction Process Problem . . . . . . . . . . . . . . . . . . . . .

Laires A. Lima, Ana I. Pereira, Clara B. Vaz, Olga Ferreira, Márcio Carocho, and Lillian Barros

Towards a High-Performance Implementation of the MCSFilter Optimization Algorithm

Leonardo Araújo, Maria F. Pacheco, José Rufino, and Florbela P. Fernandes

On the Performance of the ORTHOMADS Algorithm on Continuous and Mixed-Integer Optimization Problems

Marie-Ange Dahito, Laurent Genest, Alessandro Maddaloni, and José Neto

A Look-Ahead Based Meta-heuristics for Optimizing Continuous

Optimization Problems

Thomas Nordli and Noureddine Bouhmala

Inverse Optimization for Warehouse Management . .

Hannu Rummukainen

Model-Agnostic Multi-objective Approach for the Evolutionary Discovery

of Mathematical Models

Alexander Hvatov, Mikhail Maslyaev, Iana S. Polonskaya, Mikhail Sarafanov, Mark Merezhnikov, and Nikolay O. Nikitin

A Simple Clustering Algorithm Based on Weighted Expected Distances ....... 86 Ana Maria A. C. Rocha, M. Fernanda P. Costa, and Edite M. G. P. Fernandes

Optimization of Wind Turbines Placement in Offshore Wind Farms: Wake Effects Concerns 102 José Baptista, Filipe Lima, and Adelaide Cerveira

A Simulation Tool for Optimizing a 3D Spray Painting System João Casanova, José Lima, and Paulo Costa 
Optimization of Glottal Onset Peak Detection Algorithm for Accurate

Jitter Measurement . . . . . . . . . . . . . . . . . . . . . . . . . . . . . . . 123

Joana Fernandes, Pedro Henrique Borghi, Diamantino Silva Freitas, and João Paulo Teixeira

Searching the Optimal Parameters of a 3D Scanner Through Particle

Swarm Optimization

João Braun, José Lima, Ana I. Pereira, Cláudia Rocha, and Paulo Costa

Optimal Sizing of a Hybrid Energy System Based on Renewable Energy

Using Evolutionary Optimization Algorithms ....................

Yahia Amoura, Ângela P. Ferreira, José Lima, and Ana I. Pereira

\section{Robotics}

Human Detector Smart Sensor for Autonomous Disinfection Mobile Robot .... Hugo Mendonça, José Lima, Paulo Costa, António Paulo Moreira, and Filipe Santos

Multiple Mobile Robots Scheduling Based on Simulated Annealing Algorithm

Diogo Matos, Pedro Costa, José Lima, and António Valente

Multi AGV Industrial Supervisory System . . . . . . . . . . . . . . . . 203 Ana Cruz, Diogo Matos, José Lima, Paulo Costa, and Pedro Costa

Dual Coulomb Counting Extended Kalman Filter for Battery SOC

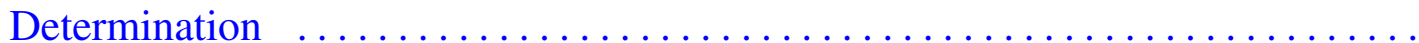

Arezki A. Chellal, José Lima, José Gonçalves, and Hicham Megnafi

Sensor Fusion for Mobile Robot Localization Using Extended Kalman

Filter, UWB ToF and ArUco Markers

Sílvia Faria, José Lima, and Paulo Costa

Deep Reinforcement Learning Applied to a Robotic Pick-and-Place

Application

Natanael Magno Gomes, Felipe N. Martins, José Lima, and Heinrich Wörtche

\section{Measurements with the Internet of Things}

An IoT Approach for Animals Tracking

Matheus Zorawski, Thadeu Brito, José Castro, João Paulo Castro, Marina Castro, and José Lima 


\title{
Dual Coulomb Counting Extended Kalman Filter for Battery SOC Determination
}

\author{
Arezki A. Chellal ${ }^{1,2(\bowtie)}(\mathbb{0})$, José Lima ${ }^{2,3}(1)$, José Gonçalves ${ }^{2,3}(\mathbb{0}$, \\ and Hicham Megnafi ${ }^{1,4}{ }_{(\mathbb{C}}$ \\ 1 Higher School of Applied Sciences, BP165, 13000 Tlemcen, Algeria \\ 2 Research Centre of Digitalization and Intelligent Robotics CeDRI, \\ Instituto Politécnico de Bragança, 5300-252 Bragança, Portugal \\ \{arezki,jllima, goncalves\}@ipb.pt \\ 3 Robotics and Intelligent Systems Research Group, INESC TEC, \\ 4200-465 Porto, Portugal \\ 4 Telecommunication Laboratory of Tlemcen LTT, University of Abou Bakr Belkaid, \\ BP119, 13000 Tlemcen, Algeria \\ h.megnafi@essa-tlemcen.dz
}

\begin{abstract}
The importance of energy storage continues to grow, whether in power generation, consumer electronics, aviation, or other systems. Therefore, energy management in batteries is becoming an increasingly crucial aspect of optimizing the overall system and must be done properly. Very few works have been found in the literature proposing the implementation of algorithms such as Extended Kalman Filter (EKF) to predict the State of Charge (SOC) in small systems such as mobile robots, where in some applications the computational power is severely lacking. To this end, this work proposes an implementation of the two algorithms mainly reported in the literature for SOC estimation, in an ATMEGA328P microcontroller-based BMS. This embedded system is designed taking into consideration the criteria already defined for such a system and adding the aspect of flexibility and ease of implementation with an average error of $5 \%$ and an energy efficiency of $94 \%$. One of the implemented algorithms performs the prediction while the other will be responsible for the monitoring.
\end{abstract}

Keywords: Prediction algorithm $\cdot$ Battery management system • Extended kalman filter · Coulomb counting algorithm · Engineering applications

\section{Introduction}

Embedded systems are ubiquitous today, but because these systems are barely perceptible, their importance and impact are often underestimated. They are used as sub-systems in a wide variety of applications for an ever-increasing

(C) Springer Nature Switzerland AG 2021

A. I. Pereira et al. (Eds.): OL2A 2021, CCIS 1488, pp. 219-234, 2021.

https://doi.org/10.1007/978-3-030-91885-9_16 
diversity of functions [1]. Whether it is a hybrid vehicle, a solar power plant, or any other everyday electrical device (PC, smartphone, drone...), the key element remains the ability to monitor, control and optimise the performance of one or more modules of these batteries, this type of device is often referred to as a Battery Management System (BMS). A BMS is one of the basic units of electrical energy storage systems, a variety of already developed algorithms can be applied to define the main states of the battery, among others: SOC, state of health $(\mathrm{SOH})$ and state of functions (SOF) that allow real-time management of the batteries.

For the BMS to provide optimal monitoring, it must operate in a noisy environment, it must be able to electrically disconnect the battery at any time, it must be cell-based and perform uniform charging and discharging across all cells in the battery [2], and the components used must be able to withstand at least the total current drawn by the load [3]. In addition, it must continuously monitor various parameters that can greatly influence the battery, such as cell temperature, cell terminal voltage and cell current. This embedded system must be able to notify the robot using the battery to either stop drawing energy from it, or to go to the nearest charging station.

However, in the field of mobile robotics and small consumer devices, such as Smarthpones or Laptops, as there are no requirements regarding the accuracy to which a BMS must be held, the standard approach such as Open Circuit Voltage (OCV) and Coulomb Counting (CC) methods are generally applied, this is mainly due to the fact that the use of more complicated estimation algorithms such as EKF, Sliding Mode and Machine Learning [4,5] requires higher computational power, thus, the most advanced battery management system algorithms reported in the literature are developed and verified by laboratory experiments using PC-based software such as MATLAB and controllers such as dSPACE. As an additional information, the most widely used battery systems in robotics today are based on electrochemical batteries, particularly lithium-ion technologies with polymer as an Electrolyte [6].

This document is devided into 5 sections, the rest of the paper is structured as follow. Section 2 promotes the work already done in this field and highlights the objectives intended throught this work. Section 3 offer a brief algorithm description implemented on the prototype. Section 4, describes the proposed solution for the system, where a block diagram and several other diagrams defining the operating principle are offered. Section 5 provides the results and offer some discussion. Finally, Sect. 5 draws together the main ideas described in this article and outlines future prospects for the development of this prototype.

\section{State of the Art Review}

Several research teams around the world have proposed different solutions to design an efficient BMS system for lithium-ion batteries. Taborelli et al. have proposed in [7], a design of an EKF and Ascending Extended Kalman Filter (AEKF) algorithms specifically developed for light vehicle categories, such as 
electric bicycles, in which a capacity prediction algorithm is also implemented tackling $\mathrm{SOH}$ estimation. The design has been validated by using simulation software and real data acquisition. In [8], Mouna et al. implemented two EKF and sliding mode algorithms in an Arduino board for SOC estimation, using an equivalent first-order battery as a model. Validation is performed by data acquisition from Matlab/Simulink. Sanguino et al. proposed in [9] an alternative design for battery system, where two batteries works alternatively, one of the batteries is charged through solar panels installed on the top of the VANTER mobile robot, while the other one provides the energy to the device. The battery SOC selection is performed following an OCV check only and the SOC monitoring is done by a coulomb counting method.

In addition, several devices are massively marketed, these products are used in different systems, from the smallest to the largest. The Battery Management System $4-15 S$ is a BMS marketed by REC, it has the usual battery protections (temperature, overcurrent, overvoltage) [10]. A cell internal DC resistance measurement technique is applied, suggesting the application of a simple resistor equivalent model and an open circuit voltage technique for SOC prediction, and an Coulomb counting technique for SOC monitoring. The device can be operated as a stand-alone unit and offers the possibility of being connected to a computer with an RS-485 for data export. This device is intended for use in solar system. Roboteq's BMS10x0 is a BMS and protection system for robotic devices developed by Roboteq, it uses a 32-bit ARM Cortex processor and offers the typical features of a BMS, along with Bluetooth compatibility for wireless states checking. It can monitor from 6 to 15 batteries at the same time. Voltage and temperature thresholds are assigned based on the chemical composition of the battery. The SOC is calculated based on an OCV and CC techniques [11].

To the best of the authors' knowledge, all research in the field of EKFbased BMS is based on bench-scale experiments using powerful softwares, such as MATLAB, for data processing. So far, the constraint of computational power limitation is not really addressed in the majority of scientific papers dealing with this subject. This paper focus on the implementation of an Extended Kalman Filter helped with a Coulomb Counting technique, called DCC-EKF, as a SOC and $\mathrm{SOH}$ estimator in ATMEGA328P microcontrollers, the proposed system is self powered, polyvalent to all types of Lithium cells, easy to plug with other systems and take into consideration most of the BMS criteria reported in the literature.

\section{Algorithm Description}

There are many methods reported in the literature that can give a representation of the actual battery charge $[4,5,7,8]$. However, these methods vary in the complexity of the implementation and the accuracy of the predicted results over long term use. There is a correlation between these two parameters, as the complexity of an algorithm increases, so does the accuracy of the results.

Also, simulation is a very common technique for evaluating and validating approaches and allows for rapid prototyping [1]; these simulations are based on 
models that are approximations of reality. MATLAB is a modeling and simulation tool based on mathematical models, provided with various tools and libraries. In this section, the two algorithms applied in the development of this prototype will be described and the results of the simulation performed for the extended Kalman filter algorithm will be given.

\subsection{Coulomb Counting Method}

The Coulomb counting method consist of a current measurement and integration in a specific period of time. The evolution of the state of charge for this method, can be described by the following expression:

$$
S O C\left(t_{n}\right)=S O C\left(t_{n-1}\right)+\frac{n_{f}}{C_{a c t u a l}} \int_{t_{n}}^{t_{n-1}} I \cdot d t
$$

where, $I$ is the current flowing by the battery, $C_{\text {actual }}$ is the actual total storable energy in the battery and $n_{f}$ represents the faradic efficiency.

Although this method is one of the most widely used methods for monitoring the condition of batteries and offers the most accurate results if properly initialized, it has several drawbacks that lead to errors. The coulomb counting method is an open-loop method and its accuracy is therefore strongly influenced by accumulated errors, which are produced by the incorrect initial determination of the faradic efficiency, the battery capacity and the SOC estimation error [12], making it very rarely applied on its own. In addition, the sampling time is critical and should be kept as low as possible, making applications where the current varies rapidly unadvisable with this method.

\subsection{Extended Kalman Filter Method}

Since 1960, Kalman Filter (KF) has been the subject of extensive research and applications, the KF Algorithm estimates the states of the system from indirect and uncertain measurements of the system's input and output. The general discrete time representation of it, is represented as follow:

$$
\left\{\begin{array}{l}
x_{k}=A_{k-1} \cdot x_{k-1}+B_{k-1} \cdot u_{k-1}+\omega_{k} \\
y_{k}=C_{k} \cdot x_{k}+D_{k} \cdot u_{k}+v_{k}
\end{array}\right.
$$

Where, $x_{k} \in R^{n \times 1}$ is the state vector, $A_{k} \in R^{n x n}$ is the system matrix in discrete time, $y_{k} \in R m \times 1$ is the output, $u_{k} \in R^{m \times 1}$ is the input, $B_{k} \in R^{n x m}$ is the input matrix in discrete time, and $C_{k} \in R^{m \times n}$ is the output matrix in discrete time. $\omega$ and $v$ represents the Gaussian distributed noise of the process and measurement.

However, Eq. 2 is only valid for linear systems, in the case of nonlinear systems such as batteries, the EKF is applied to include the non-linear behaviour and to determine the states of the system [14]. The EKF equation has the following form:

$$
\left\{\begin{aligned}
x_{k+1} & =f\left(x_{k}, u k\right)+\omega_{k} \\
y_{k} & =h\left(x_{k}, u k\right)+v_{k}
\end{aligned}\right.
$$


Where,

$$
A_{k}=\frac{\partial f\left(x_{k}, u_{k}\right)}{\partial x_{k}}, \quad C_{k}=\frac{\partial h\left(x_{k}, u_{k}\right)}{\partial x_{k}}
$$

After initialization, the EKF Algorithm always goes through two steps, prediction and correction. The KF is extensively detailed in many references [13-15], we will therefore not focus on its detailed description. The Algorithm 1 summarize the EKF function implemented in each Slave ATMEGA328P microcontroller, the matrices $A_{k}, B_{k}, C_{k}, D_{k}, x_{k}$ are discussed in Sect. 3.3.

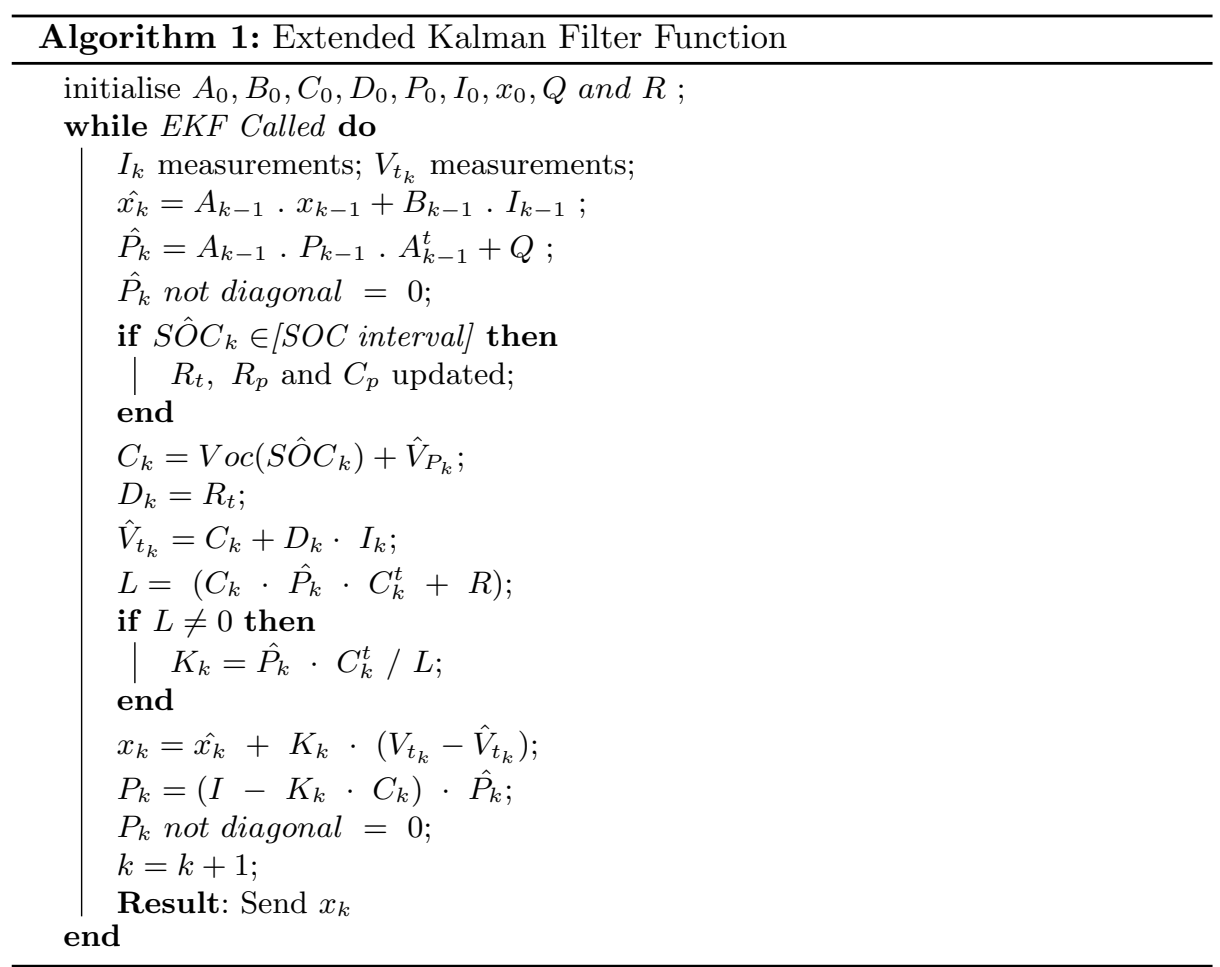

The overall performance of the filters is set by the covariance matrix $\mathrm{P}$, the process noise matrix $\mathrm{Q}$ and the measurement noise $\mathrm{R}$. The choice of these parameters were defined throught experience and empirical experiment and are given by:

$$
Q=\left[\begin{array}{cc}
0.25 & 0 \\
0 & 0
\end{array}\right], P=\left[\begin{array}{ll}
1 & 0 \\
0 & 1
\end{array}\right] \text { and } R=0.00001
$$

Data obtained from discharge tests of different cells simulations were applied to verify the accuracy of this estimator with known parameters and are reported in Sect. 3.4. The EKF Algorithm implemented in the ATMEGA328P microcontrollers has a sampling time of $0.04 \mathrm{~s}$. 


\subsection{Battery Modelling}

Two battery models are most often applied, the Electrochemistry model, which gives a good representation of the internal dynamics of the batteries, and the Electrical circuit model, which allows the behaviour of the batteries to be translated into an electrical model and can be easily formulated into mathematical formula. For this study, the second model, considered more suitable for use with a microcontroller, is chosen. The most commonly applied electrical model uses a simple RC equivalent battery circuit model, with two resistors $\left(R_{p}\right.$ and $\left.R_{t}\right)$, a capacitor $\left(C_{p}\right)$, a voltage source $\left(V_{o c}\right)$ and a current flowing through it $(I)$, as shown in Fig. 1. It represents the best trade-off between accuracy and complexity.

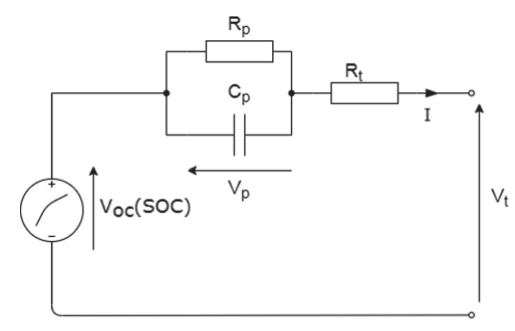

Fig. 1. First order equivalent battery model

The terminal voltage denoted as $V_{t}$, represents the output voltage of the cell, and is given by Eq. 5 .

$$
V_{t}=V_{o c}(S O C)+I \cdot R_{t}+V_{p}
$$

A non-linear relationship exist between $S O C$ and $V_{o c}$, a representation employing the seventh-order polynomial to fit the overall curve can be expressed and is described with the following relation:

$$
\begin{aligned}
& V_{o c}(S O C)=3.4624 e^{-12} \cdot S O C^{7}-1.3014 e^{-9} \cdot S O C^{6}+1.9811 e^{-7} \cdot S O C^{5} \\
& \quad-1.5726 e^{-5} \cdot S O C^{4}+6.9733 e^{-4} \cdot S O C^{3}-0.017 \cdot S O C^{2}+0.21 \cdot S O C+2.7066
\end{aligned}
$$

The time derivative of Eq. 1 can be formulated as given in Eq. 7,

$$
S \dot{O C}=\frac{I}{C_{\text {actual }}}
$$

The polarization voltage is given as,

$$
\dot{V}_{p}=-\frac{1}{R_{p} C_{p}} V_{p}+\frac{1}{C_{p}} I
$$


From the Eqs. 5, 7 and 8, it is possible to deduce the equation characterising the behaviour of the battery, which is expressed as the following system:

$$
\left\{\begin{aligned}
{\left[\begin{array}{c}
S \dot{O} C \\
\dot{V}_{p}
\end{array}\right] } & =\left[\begin{array}{cc}
0 & 0 \\
0 & \frac{-1}{R_{p} C_{p}}
\end{array}\right] \cdot\left[\begin{array}{c}
S O C \\
V_{p}
\end{array}\right]+\left[\begin{array}{c}
\frac{1}{C_{\text {actual }}} \\
\frac{C_{p}}{C_{p}}
\end{array}\right] \cdot I \\
V_{t} & =\left[\begin{array}{ll}
\text { poly } & 1
\end{array}\right] \cdot\left[\begin{array}{c}
S O C \\
V_{p}
\end{array}\right]+R_{t} \cdot I
\end{aligned}\right.
$$

Where, poly is the polynomial formula described in Eq. 6. Since the microcontroller processes actions in discrete events, the previous system must be transcribed into its discrete form, which is written as follows,

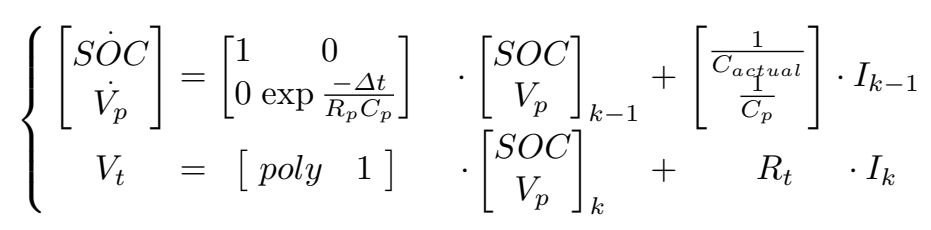

From both Eqs. 2 and 10, it is deduced that the current $I$ is the input of the system, the voltage $V_{t}$ is the output and the matrices $A_{k}, B_{k}, C_{k}$ and $D_{k}$ matrices are given by:

$$
A_{k}=\left[\begin{array}{ll}
1 & 0 \\
0 & \exp \frac{-\Delta t}{R_{p} C_{p}}
\end{array}\right], \quad B_{k}=\left[\begin{array}{c}
\frac{1}{C_{a c \text { ual }}} \\
\overline{C_{p}}
\end{array}\right], x_{k}=\left[\begin{array}{c}
S O C_{k} \\
V_{P_{k}}
\end{array}\right], \quad C_{k}^{t}=\left[\begin{array}{c}
\text { poly } \\
1
\end{array}\right], \quad D_{k}=R_{t}
$$

\subsection{Simulation Results}

Given the large number of articles dealing with the validation of these algorithms $[13,16,17]$, it is however not necessary to dwell on this subject. Nevertheless, a simulation of the algorithm is proposed in this section, in order to confirm the good follow-up of the algorithm according to the chosen parameters.

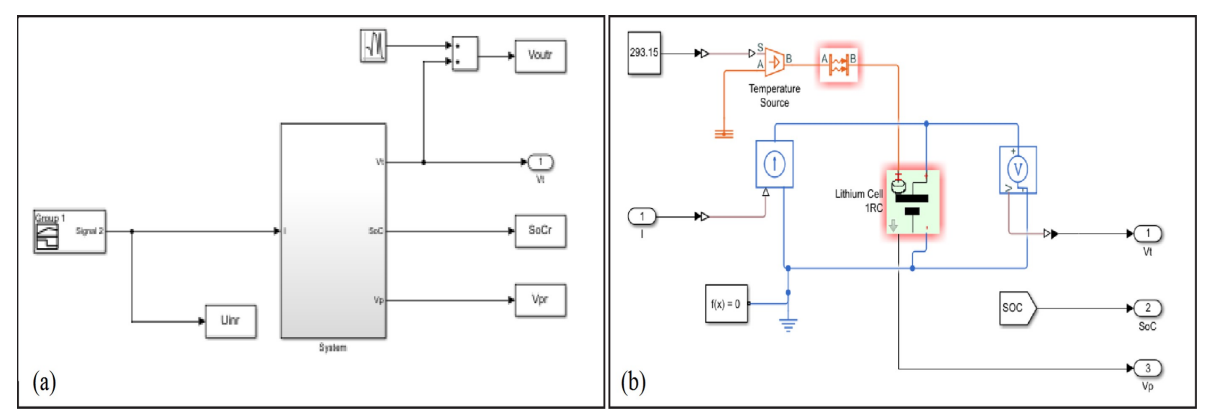

Fig. 2. (a) overall Simulink model highlighting the Input/Output of the simulation (b) Battery sub-system model 
The graphical representation of MATLAB/Simulink using graphical blocks to represent mathematical and logical constructs and process flow is intuitive and provides a clear focus on control and observation functions. Although Simulink has been the tool of choice for much of the control industry for many years [18], it is best to use Simulink only for modelling battery behaviour and not for the prediction algorithm. The battery model presented before is applied as a base model, the more complex model will not be able to simulate all possible cases and will therefore increase the time needed for development. Figure 2 represents the battery simulation used in MATLAB/Simulink.

Figure 3 shows a comparison of the state of charge of the battery with simulation for the Extended Kalman filter, and a plot of the error with the real and the predicted one. The test consists of a $180 \mathrm{~s}$ discharge period followed by a one hour rest period, the cell starts fully charged and the EKF state of charge indicator is set at $50 \%$ SOC.
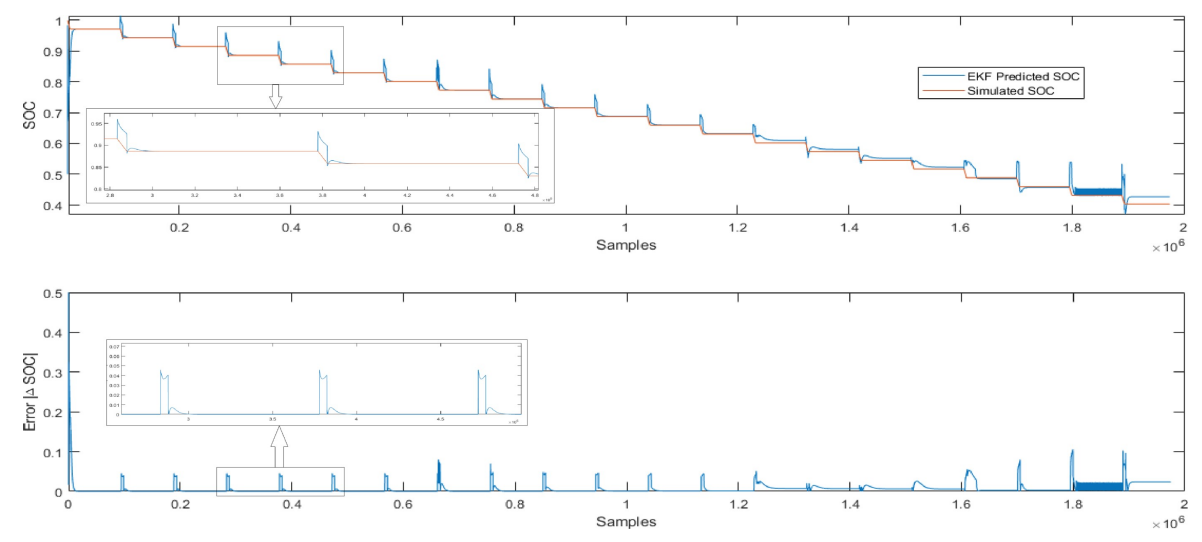

Fig. 3. Estimated and reference SOC comparison with the absolute error of the SOC for Extended Kalman Filter with a constant discharge test and a rest period.

The continuous updating of the correction gain $(K)$, which is due to the continuous re-transcription of the error covariance matrices, limits the divergence as long as the SOC remains within the predefined operating range (80\%-40\%). The response of the observer to the determination of the SOC can be very fast, on the order of a few seconds. Also, from the previous figure it is clear that, for known parameters, the SOC can be tracked accurately with less than $5 \%$ of error, and record a perfect prediction for when the battery is at rest. Figure 4 shows the simulation results of a constant current discharge test.

Although a good SOC monitoring by the EKF, it still requires a good determination of the battery parameter. Some reported works $[19,20]$, have proposed the use simple algebraic method or a Dual Extended Kalman Filter (DEKF) as an online parameter estimator, to estimate them on the fly. Unfortunately, these methods require a lot of computational power, to achieve a low sampling time 

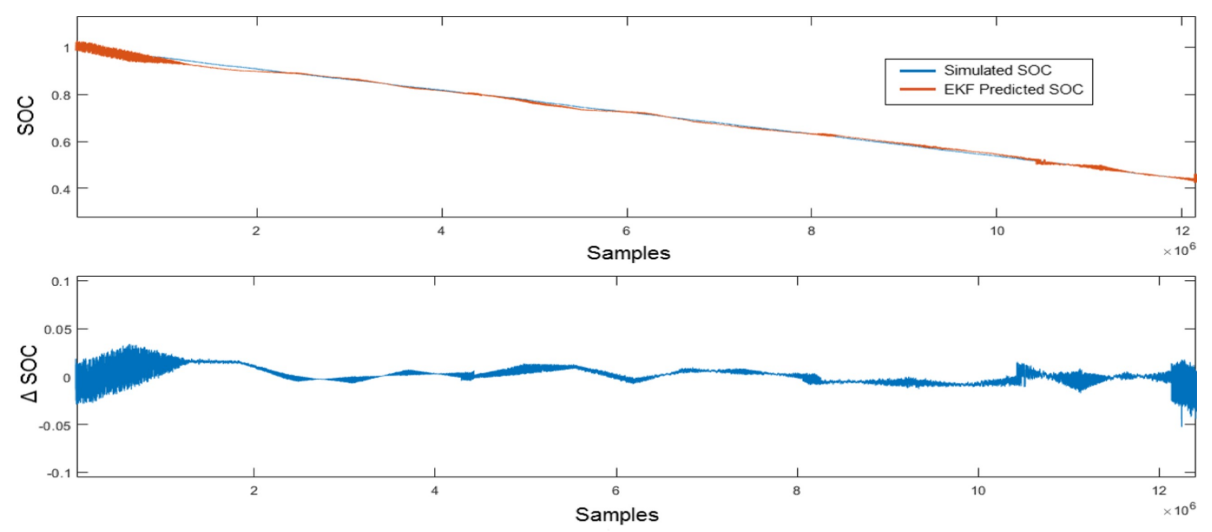

Fig. 4. Estimated and reference SOC comparison with the absolute error of the SOC for Extended Kalman Filter with a constant discharge test.

and reach convergence, which is difficult to achieve with a simple ATMEGA328P. For this reason, and given the speed at which the EKF is able to determine the battery SOC at rest, the EKF algorithm is applied to determine the battery SOC at start-up and then the $\mathrm{CC}$ algorithm is applied to carry on the estimation.

\section{Proposed Solution}

The system is designed to be "Plug and Play", with two energy input and output located on one side only, future users of the product can quickly install and initialize the BMS, which works for all types of Lithium Model 18650 batteries, the initialization part is described in more depth in the next section. The connecting principle of the electronic components is illustrated in Fig. 5.

\subsection{Module Connection}

The Master microcontroller is the central element of the system, the ATMEL's ATMEGA328P was chosen for its operational character and low purchase cost, it collect the State of Charge predicted by each ATMEGA328P slave microcontroller and control the energy flow according to the data collected. This microcontroller is connected to push buttons and an OLED screen to facilitate communication with the user. Power is supplied directly from the batteries, the voltage is stabilised and regulated for the BMS electronics by an step down voltage regulator.

The Inter-Integrated Circuit (I2C) is a bus interface connection using only 2 pins for data transfer, it is incorporated into many devices and is ideal for attaching low-speed peripherals to a motherboard or embedded system over a short distance, it provides a connection-oriented communication with acknowledge [21]. Figure 6 represent the electronic circuit schematic diagram. 


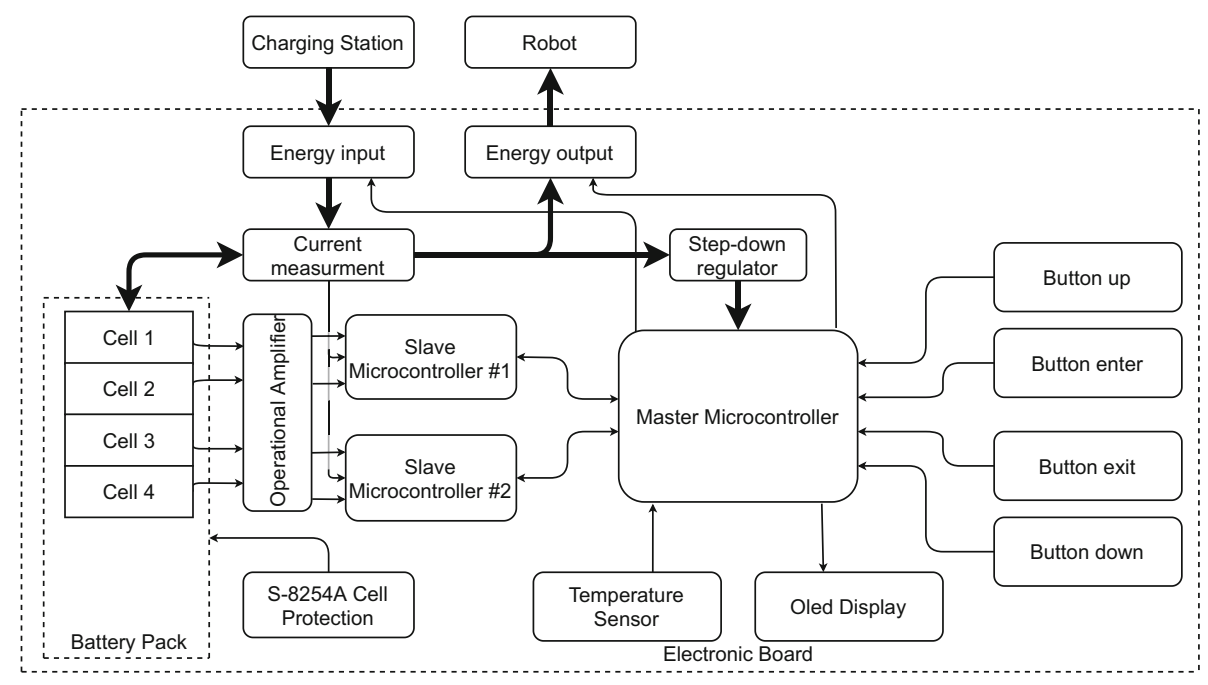

Fig. 5. Block diagram of the system

The 18650 Lithium cells are placed in the two cell holder $(1 \mathrm{BH}, 2 \mathrm{BH})$. The Oled display (OLED1) is connected via I2C by the Serial Clock and Serial DATA line, called SCL and SDA respectively, the ATMEGA328P microcontroller (MASTER, SLAVE1, SLAVE2) are connected to the I2C wire through pins PC5 and PD4. The current is measured from the ACS712 (U1) and gathered by the slave microcontrollers (SLAVE1, SLAVE2) from the analog pin PC0. A quadruple precision amplifier LM324 (U5) is used to measure the voltage at each cell terminal, with the use of a simple voltage divider. The push buttons are linked to the master microcontroller via the digital pins (PD2, PD3, PB4, PB5), an IRF1405 N-Channel Mosfet is used as a power switch to the load (mobile robot) while an IRF9610 P-Channel Mosfet is used as a power switch from the power supply, they are controlled from master ATMEGA328P pins PB3 and PB2 respectively. A step down voltage regulator is applied to regulate the voltage supplied to the electronics to $5 \mathrm{~V}$, this power is either gathered from the battery pack or the power supply.

\subsection{Operating Principle}

This system is mainly characterised by two modes, the initialisation mode and the on-mode, these two modes are very dependent on each other and it is possible to switch from one mode to the other at any time using a single button. Figure 7 briefly summarizes the operation principale for both modes. 


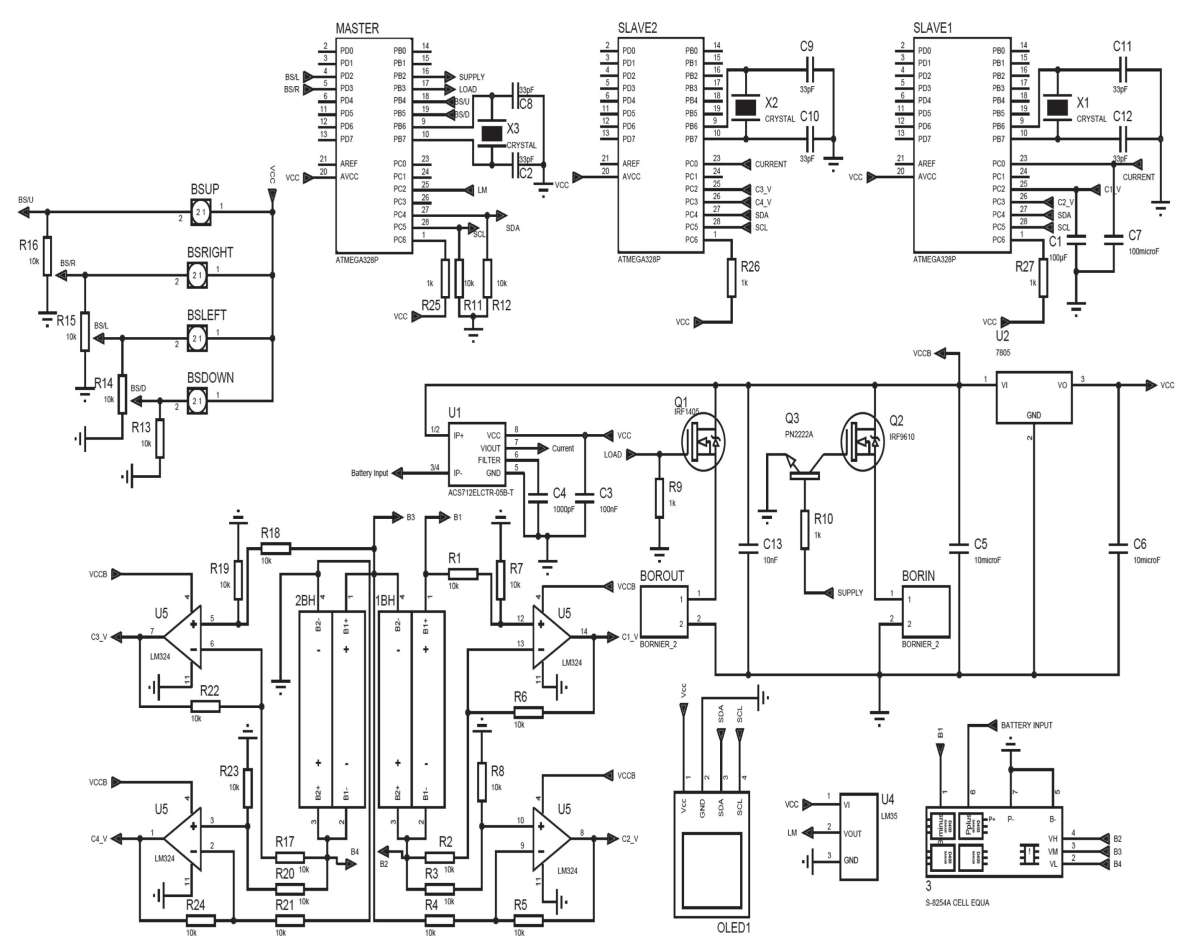

Fig. 6. Schematic diagram of the electronic circuit

Because the characteristics between the batteries are not uniform, and can vary greatly, and in order to achieve high accuracy of SOC prediction, the initialization mode is introduced into the system, it is possible to skip this mode and ignore it, but at the cost of reduced accuracy. Through this mode it is possible to enter the capacity of the batteries installed, the actual SOC to ensure a more accurate result, as well as to activate an additional protection to limit further the discharges and the use or not of the EKF algorithm.

As for the on mode, according to what was initialize, the BMS begins by carrying out the assigned tasks for each battery cell in parallel.

- If the EKF prediction was set on during the initialization mode, the device shall begin the prediction of the actual SOC value for each cell, and prohibits the passage of current other than that which powers the BMS for a period of approximately 3 min (Fig. 7).

- If the EKF prediction was set off during the initialization mode, and an SOC Value was introduced, the device allows the current to flow directly, and start the SOC monitoring according to the chosen value.

- If the Protection was set off, the battery will be discharged and charged even deeper, taking the risk of shorten the life span of the batteries. 


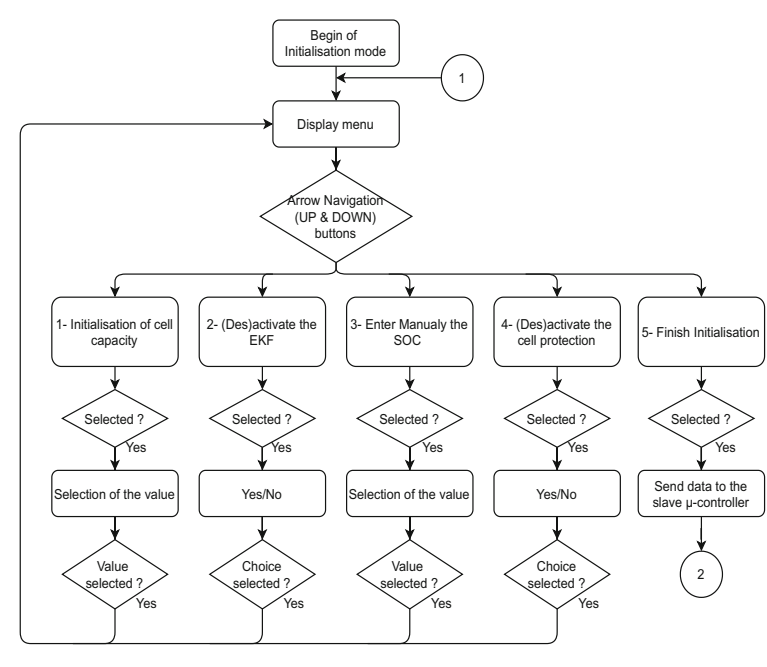

(a)

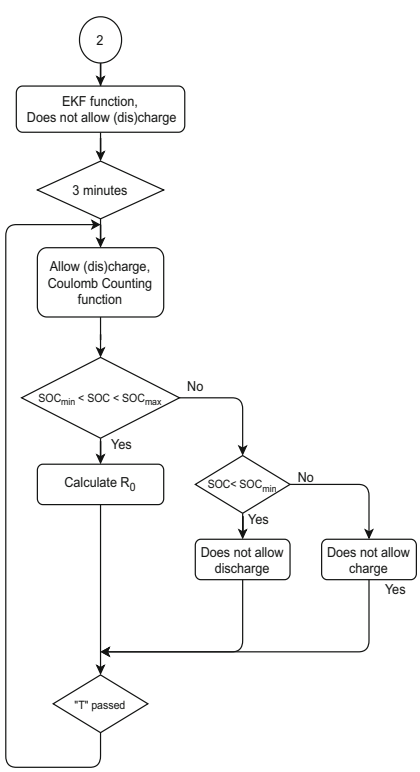

(b)

Fig. 7. Flow chart of the system algorithm. (a) Initialisation mode flow chart. (b) On-mode flow chart with EKF algorithm activated.

The battery states are determined for each cell independently of the others by a specific microcontroller assigned to it, and works with the principle of Master/Slave communication. In order to keep a low sampling time, one slave microcontroller is assigned to two batteries, reaching a sampling time of $0.085 \mathrm{~s}$.

\section{$5 \quad$ Results and Discussions}

The result is the design of a BMS that takes into account the already established requirements for this type of system and defines new ones, such as flexibility and size, that must be met for mobile robots. A breadboard conception was first carried out to perform the first tests. After power up, the initialisation mode is first started as expected. After that, while the on-mode is running, the voltage, current and SOC data are displayed on the screen, an accuracy of $100 \mathrm{mV}$ and $40 \mathrm{~mA}$ is achieved. When the cell is at rest and for a $S O C \in[80 \%--40 \%]$, the EKF prediction reaches an error of $5 \%$ in the worst case, for SOC cells above $80 \%$ the accuracy drops to $8 \%$, while for SOC below $30 \%$ the algorithm diverges completely (Fig. 10). These results are quite understandable, as the linearization of these curves is done in only 10 points. A cell protection is performed by an external circuit based on a S-8254A, it includes a high accuracy voltage detection for lithium battery, avoiding over voltage while charging the batteries, they are widely applied in rechargeable battery pack, due to their low costs and good caracteristics [22]. 
The total storable power offered by this model varies according to the capacity of the installed cells. It is clear that the use of 4 lithium cells of $2200 \mathrm{mAh}$, gives the system a battery capacity of $8800 \mathrm{mAh}$ which is sufficient in the majority of small robots application, it should be noted that it is also possible to implement the $5500 \mathrm{mAh}$ cell, giving the product developed great flexibility by offering the possibility of choosing the battery capacity to users. The maximum current that can be delivered is $2 \mathrm{~A}$, and therefore allows to offer a power up to $32 \mathrm{~W}$, which is sufficient to power the electronics of the robot, beyond this value and in order to offer an optimal safety to the battery, a shutdown protocol will stop the discharge of the battery. As for the charging process, the maximum current supplied by an external source is currently $0.5 \mathrm{~A}$, in order to have a good protection when charging the batteries. With such a current, the charging process is slow, in fact it will take $8 \mathrm{~h}$ and a half to reach the $80 \%$ SOC advised for $4 \times 2200 \mathrm{mAh}$ cells. Figure 8 shows the breadboard circuit.

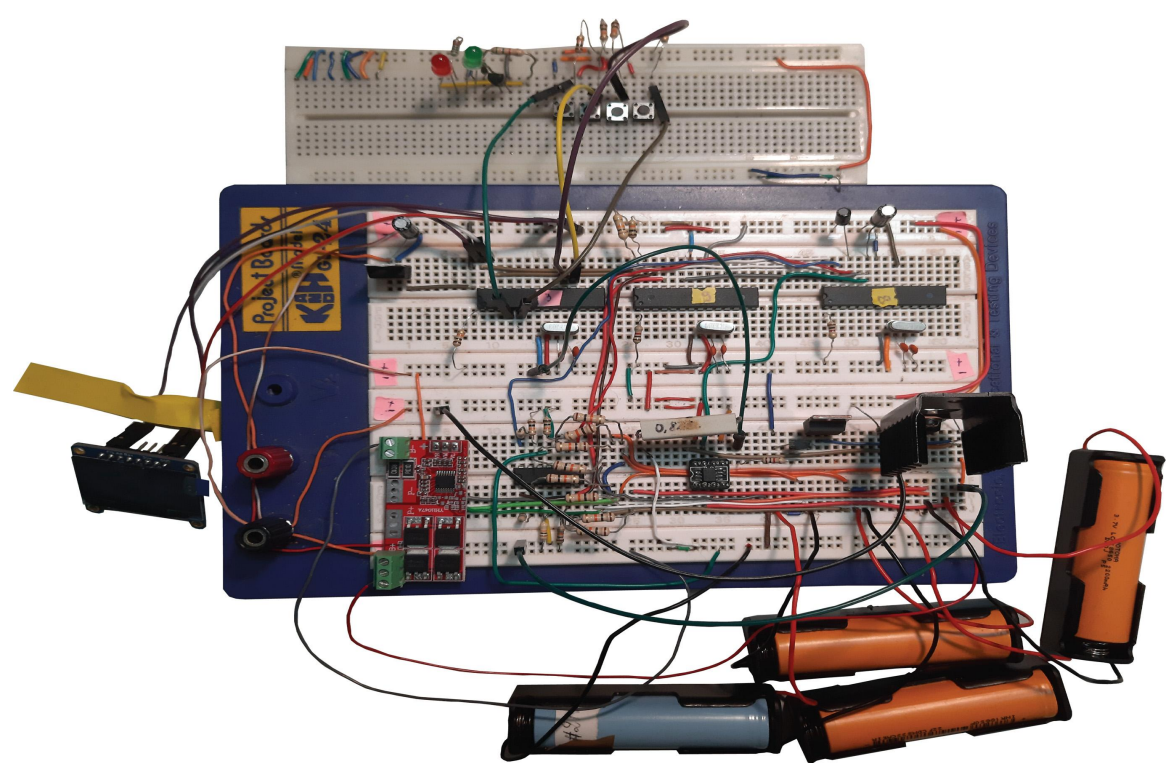

Fig. 8. Breadboard circuit

The BMS electronics consume in average $1.15 \mathrm{~W}$, giving the product an efficiency of $96.15 \%$. While charging the battery, and because a constant charging power is set, the IRF9610 Mosfet leads to a constant loss of power of $0.52 \mathrm{~W}$, the efficiency then reaches $90.38 \%$. For discharging the battery, as the regulation of the voltage supplied to the robot is left to the user and is done with a variable current, it is difficult to define with precision the efficiency of this device, it can however reach $2.5 \%$, which makes the total efficiency to be $93.60 \%$. Thus, in general the average efficiency of this product is $94.37 \%$. Figure 9 shows a printed circuit representation of the proposed prototype. 

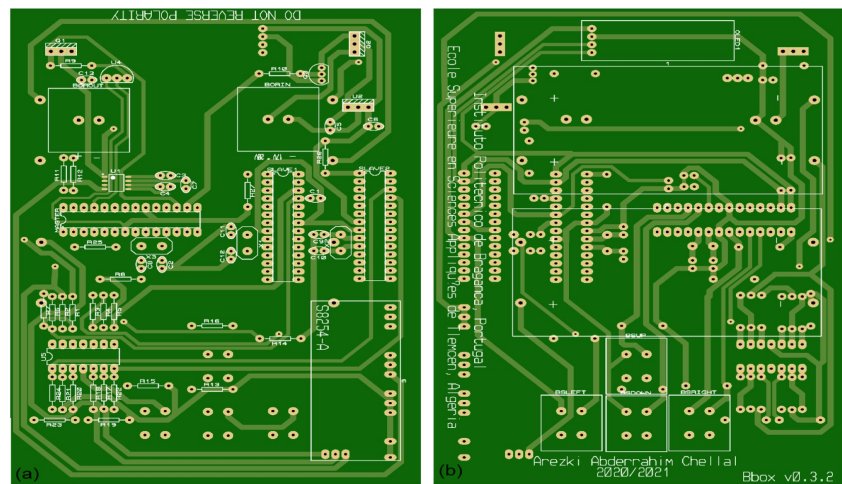

Fig. 9. Printed Cirduit Board of the prototype. (a) componants view. (b) top view.

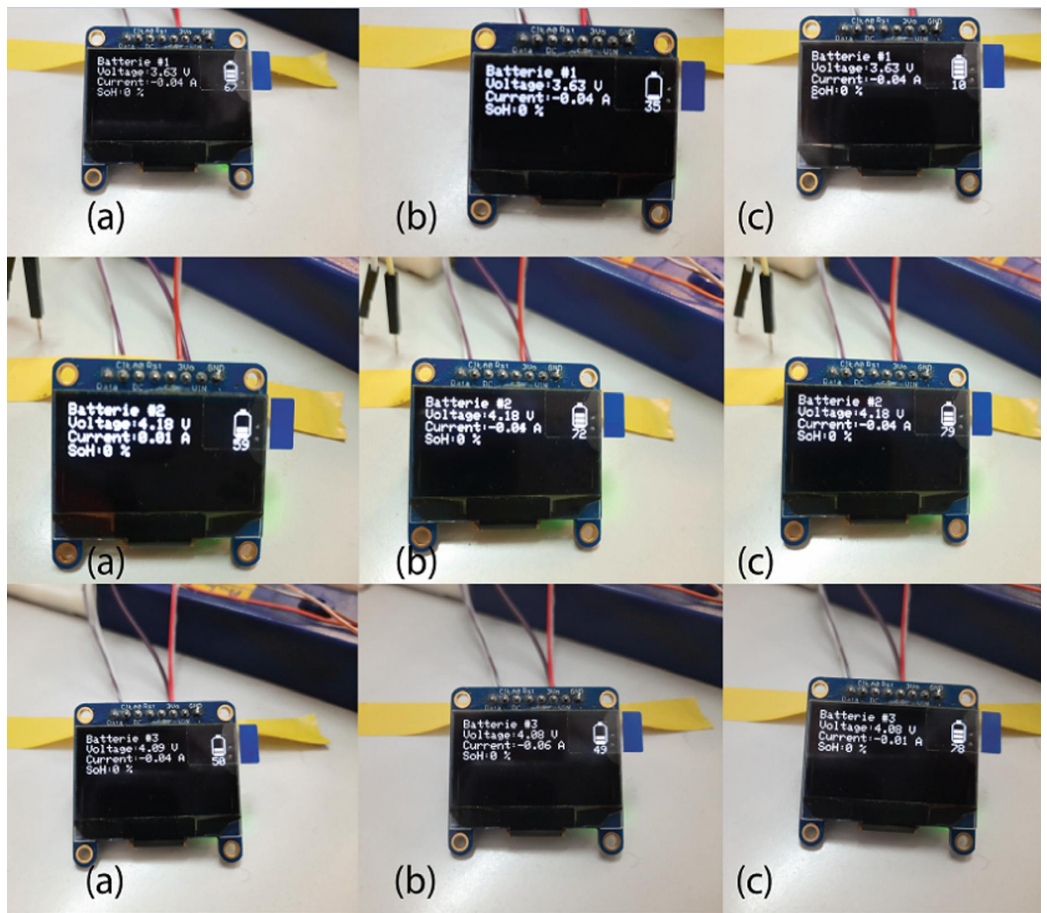

Fig. 10. On-mode display of cells 1, 2 and 3 with a SOC value of $9.51 \%, 77.25 \%$ and $71.10 \%$ respectively. (a) Average SOC of the first 50 iteration. (b) Few seconds after. (c) approximately $1 \mathrm{~min}$ after. For cell 1, the algorithm diverges for the SOC estimate. For cell 2, the estimate has reached the SOC value with an error of $1.75 \%$. For cell 3 , after $1 \mathrm{~min}$, the prediction has not yet reached the estimated value with an error of $6.90 \%$. 
The SOC values, voltage and current are displayed directly on the Oled screen as shown in Fig. 10. It is possible to display the overall battery status, or cell by cell for a more detailed view. Taking into account that this approach and the algorithm are constantly being improved, not all features such as the $\mathrm{SOH}$ and temperature are fully integrated.

\section{Conclusion and Future Work}

This paper proposes the DCC-EKF approach where the state of charge is first predicted using an EKF algorithm, after which the SOC is given to the Columbus measurement algorithm to monitor this quantity. Most commercial products only use the OCV to initially predict the SOC, a technique that is widely implemented, but is severely inaccurate, especially when the BMS is powered directly from the batteries, so the batteries never reach a resting state. The balancing and overvoltage protection of the BMS is provided by an external circuit based on the S-8245A module. The proposed BMS is small $(10 \mathrm{~cm} \times 15 \mathrm{~cm})$, easy to use and has shown accurate SOC prediction with an error of $5 \%$ and good performance in noisy operation. The prototype also offers a good efficiency of about $93 \%$.

Future developments will focus on implementing a more accurate state of charge prediction algorithm, such as DEKF and AEKF, as well as a more accurate health prediction algorithm, while aiming to make it more user-friendly and improve the user experience. The temperature effect will also be addressed in future versions, as it is actually neglected, as well as the increase of the product efficiency. In addition, it is considered to Implement a Learning Algorithm, to further enhance the prediction accuracy, with a fast charging feature.

\section{References}

1. Marwedel, P.: Embedded Systems Foundations of Cyber-Physical Systems, and the Internet of Things. Springer Nature (2021)

2. Park, K.-H., Kim, C.-H., Cho, H.-K., Seo, J.-K.: Design considerations of a lithium ion battery management system (BMS) for the STSAT-3 satellite. J. Power Electron. 10(2), 210-217 (2010)

3. Megnafi, H., Chellal, A.-A., Benhanifia, A.: Flexible and automated watering system using solar energy. In: International Conference in Artificial Intelligence in Renewable Energetic Systems, pp. 747-755. Springer, Cham, Tipaza (2020). https://doi.org/10.1007/978-3-030-63846-7_71

4. Xia, B., Lao, Z., Zhang, R., et al.: Online parameter identification and state of charge estimation of lithium-ion batteries based on forgetting factor recursive least squares and nonlinear Kalman filter. Energies 11(1), 3 (2018)

5. Hannan, M.A., Lipu, M.H., Hussain, A., et al.: Toward enhanced State of charge estimation of Lithium-ion Batteries Using optimized Machine Learning techniques. Sci. Rep. 10(1), 1-15 (2020)

6. Thomas, B.-R.: Linden's Handbook of Batteries, 4th edn. McGraw-Hill Education, New York (2011) 
7. Taborelli, C., Onori, S., Maes, S., et al.: Advanced battery management system design for SOC/SOH estimation for e-bikes applications. Int. J. Powertrains 5(4), 325-357 (2016)

8. Mouna, A., Abdelilah, B., M'Sirdi, N.-K.: Estimation of the state of charge of the battery using EKF and sliding mode observer in Matlab-Arduino/LabView. In: 4th International Conference on Optimization and Applications, pp. 1-6. IEEE, Morocco (2018)

9. Sanguino, T.-D.-J.-M., Ramos, J.-E.-G.: Smart host microcontroller for optimal battery charging in a solar-powered robotic vehicle. IEEE/ASME Trans. Mechatron. 18(3), 1039-1049 (2012)

10. REC-BMS.: Battery Management System 4-15S. REC, Control your power, Slovenia (2017)

11. Robote, Q.: BMS10x0, B40/60V, 100 Amps Management System for Lithium Ion Batteries. RoboteQ, USA (2018)

12. Kim, I.-S.: A technique for estimating the state of health of lithium batteries through a dual-sliding-mode observer. IEEE Trans. Power Electron. 25(4), 10131022 (2009)

13. Mastali, M., Vazquez-Arenas, J., Fraser, R.: Battery state of the charge estimation using Kalman filtering. J. Power Sources 239, 294-307 (2013)

14. Campestrini, C., Heil, T., Kosch, S., Jossen, A.: A comparative study and review of different Kalman filters by applying an enhanced validation method. J. Energ. Storage 8, 142-159 (2016)

15. Bishop, G., Welch, G.: An introduction to the kalman filter. In: Proceedings of SIGGRAPH, Course. Proceedings of SIGGRAPH, vol. 41, pp. 27599-23175 (2001)

16. Campestrini, C., Horsche, M.-F., Zilberman, I.: Validation and benchmark methods for battery management system functionalities: state of charge estimation algorithms. J. Energ. Storage 7, 38-51 (2016)

17. Yuan, S., Wu, H., Yin, C.: State of charge estimation using the extended Kalman filter for battery management systems based on the ARX battery model. Energies 6(1), 444-470 (2013)

18. Marian, N., Ma, Y.: Translation of simulink models to component-based software models. In: 8th International Workshop on Research and Education in Mechatronics, pp. 262-267. Citeseer, Location (2007)

19. Hu, T., Zanchi, B., Zhao, J.: Determining battery parameters by simple algebraic method. In: Proceedings of the 2011 American Control Conference, pp. 3090-3095. IEEE, San Francisco (2011)

20. Xu, Y., Hu, M., Zhou, A., et al.: State of charge estimation for lithium-ion batteries based on adaptive dual Kalman filter. Appl. Math. Modell. 77(5), 1255-1272 (2020)

21. Mazidi, M.-A., Naimi, S., Naimi, S.: AVR Microcontroller and Embedded Systems. Pearson, India (2010)

22. ABLIC INC.: S-8254A Series Battery Protection IC for 3-Serial- or 4-Serial-CELL Pack REV.5.2. ABLIC, Japan (2016)

23. Chatzakis, J., Kalaitzakis, K., Voulgaris, C., Manias, N.-S.: Designing a new generalized battery management system. IEEE Trans. Ind. Electron. 50(5), 990-999 (2003)

24. Chen, L., Xu, L., Wang, R.: State of charge estimation for lithium-ion battery by using dual square root cubature kalman filter. Mathematical Problems in Engineering 2017 (2017) 